\title{
Sémiotique et rhétorique dans deux contes de
} Machado de Assis

Semiotics and Rhetoric in two short tales by Machado de Assis

\section{Glaucia Muniz Proença Lara et Dilson Ferreira Cruz}

Traducteur : Jean Briant

\section{(2) OpenEdition} Journals

\section{Édition électronique}

URL : http://journals.openedition.org/aad/1152

DOI : 10.4000/aad. 1152

ISSN : 1565-8961

\section{Éditeur}

Université de Tel-Aviv

\section{Référence électronique}

Glaucia Muniz Proença Lara et Dilson Ferreira Cruz, « Sémiotique et rhétorique dans deux contes de Machado de Assis », Argumentation et Analyse du Discours [En ligne], 7| 2011, mis en ligne le 15 octobre 2011, consulté le 23 septembre 2019. URL : http://journals.openedition.org/aad/1152 ; DOI : 10.4000/aad. 1152

Ce document a été généré automatiquement le 23 septembre 2019.

\section{cc) $($ ) $\Theta$}

Argumentation \& analyse du discours est mis à disposition selon les termes de la licence Creative Commons Attribution - Pas d'Utilisation Commerciale - Pas de Modification 4.0 International. 


\title{
Sémiotique et rhétorique dans deux contes de Machado de Assis
}

\author{
Semiotics and Rhetoric in two short tales by Machado de Assis \\ Glaucia Muniz Proença Lara et Dilson Ferreira Cruz \\ Traduction : Jean Briant
}

\section{Introduction}

1 Cet article se propose d'établir un dialogue entre la sémiotique greimassienne, considérée dans ses lignes générales comme théorie de la signification, et la rhétorique qui, dans l'acception aristotélicienne, se préoccupe essentiellement de découvrir ce qui peut engendrer la persuasion ${ }^{1}$. Barros (2008: 27) signale le caractère productif de l'articulation entre ces deux disciplines, que ce soit dans l'examen des figures de contenu et d'expression, ou dans le traitement des questions discursives de persuasion et d'argumentation - les contrats fiduciaires, l'interaction entre sujets et la construction de l'identité ou de l'ethos - aspect que nous privilégierons ici.

2 C'est dans cette perspective que nous examinerons deux contes de l'écrivain brésilien Joaquim Maria Machado de Assis (1839-1908) publiés dans Papéis Avulsos ( $1^{\mathrm{e}}$ édition: 1882), sous les titres de : Teoria do medalhão (Théorie du médaillon) et 0 espelho (Le miroir) ${ }^{2}$. Bien que différents, ces deux contes ont un point commun : l'un et l'autre débattent du processus de déconstruction de l'identité. Dans Théorie du médaillon nous est proposée une théorie que nous pourrions dénommer rhétorique de l'inanité, puisqu'elle se propose de construire un comportement et un discours vides, donc dépourvus d'identité. Dans Le miroir, nous assistons à un processus involontaire de perte d'identité qui, par delà les aspects discursifs, tend à s'approprier entièrement le sujet, jusqu'à menacer son existence.

3 En réaffirmant la profonde différence entre les deux contes choisis - malgré leur point commun, la "déconstruction de l'identité " -, nous chercherons à mettre en évidence ce qui, dans chacun d'eux, constitue ses traits les plus saillants. Ainsi, dans Théorie du 
médaillon nous insisterons sur les faire persuasif et interprétatif qui s'instaurent soit dans l'instance du texte-énoncé : celle des acteurs ou des personnages qui participent à la trame du récit, soit dans l'instance de l'énonciation: celle de l'énonciateur et de l'énonciataire (l'auteur et le lecteur implicites) qui, par le truchement du texte, se mettent en rapport. Dans Le miroir, on se penchera sur l'ethos du personnage central tel qu'il se (dé)construit au long du texte.

Nous insistons sur le fait qu'en raison des différences que présentent ces deux contes, les analyses auront des points de départ distincts et suivront des chemins également distincts. La problématique de l'identité n'en sera pas moins, comme nous l'avons déjà indiqué, au centre des deux analyses : en effet, dans les deux cas se manifeste la nonviabilité de cette identité, ce qui renforce l'une des caractéristiques de l'œuvre de Machado de Assis : la nature scindée de l'homme moderne, incapable d'être unique et en conséquence de conserver son intégrité.

\section{La persuasion dans Théorie du médaillon}

Ce conte est un dialogue entre un père (dont on ne nous donne pas le nom) et son fils (appelé Janjão ${ }^{3}$ ), le jour où celui-ci accède à la majorité, et consiste dans un ensemble d'instructions que le père, dans son faire persuasif/discursif, transmet à son fils pour que, dans le faire interprétatif qui lui revient, il veuille et/ou doive exercer le «noble office » de médaillon ${ }^{4}$, c'est-à-dire atteindre au prestige dans une société d'apparences. Les conseils se résument en réalité à des comportements médiocres qui, une fois assumés, permettront au fils, sans qu'il lui en coûte de grands efforts, de monter dans l'échelle sociale.

6 Rappelons que la théorie sémiotique cherche à expliquer non seulement ce que dit le texte mais, surtout, comment il fait pour dire ce qu'il dit, en examinant le plan du contenu au moyen du parcours génératif de sens, qui va du plus simple et du plus abstrait - le niveau fondamental - au plus complexe et au plus concret - le niveau discursif -, en passant par un niveau intermédiaire - le narratif.

7 Ainsi, au niveau narratif, si nous considérons les quatre programmes (PNs) ${ }^{5}-$ manipulation, compétence, performance et sanction - qui, intégrés en parcours, forment le schéma narratif canonique, nous constatons que le conte se résume pratiquement à la manipulation opérée par le destinateur-père sur le destinataire-fils et à la tentative que le premier fait, au moyen des conseils qu'il présente, de doter le second de la compétence nécessaire à l'action (performance), d'autant plus que Janjão a déjà une propension naturelle à la non-production d'idées, comme le lui fait remarquer son père : «-Si je ne m'abuse, mon fils, tu me parais doué de la parfaite ineptie mentale qui convient à ce noble office » (45).

8 En somme, au moyen de son faire persuasif, le père (destinateur-manipulateur) se propose non seulement d'amener son fils à vouloir et/ou à devoir être un médaillon manipulation proprement dite -, mais aussi de le doter du savoir et du pouvoir de le faire - compétence modale du sujet qui, dans le cas présent, a déjà une espèce de prédisposition ou de "savoir » inné : son « ineptie » mentale, qui devra toutefois être perfectionnée à partir des connaissances et de l'expérience du père, lui-même médaillon frustré, paradoxalement, pour n'avoir pas eu un père qui l'instruise. 
Cependant, la manipulation ne sera réussie que si le système de valeurs sur lequel elle se fonde est partagé à la fois par le manipulateur et par le manipulé et à condition qu'il y ait entre eux une certaine complicité, ce qui implique donc un accord sur la "vérité » des valeurs en jeu. Dans cette perspective, le destinataire de la manipulation - le fils pour accepter le contrat proposé par le destinateur-manipulateur (le père) doit croirevrai et savoir-fondé le discours qui lui est présenté, les valeurs (associées à l'office de médaillon) qu'il véhicule et l'instance même qui profère le discours (en l'occurrence, l'instance paternelle avec les attributs qui lui sont inhérents). Dans le cas en question, ce qui intéresse est ce qui paraît-vrai et qui, étant présenté comme vrai dans et par le discours, est pris comme tel par le sujet de la manipulation: la confiance dans les paroles sur les choses et sur le monde devenant croyance dans les choses mêmes et dans le monde (Greimas, 1983 : 17). Nous voyons donc que le faire-croire implique un faire paraître vrai, ce qui reprend la question de la vraisemblance si chère à la rhétorique.

10 Comme l'affirme Cruz (2009: 41), la préoccupation centrale de la rhétorique n'est pas de découvrir la vérité (ontologique), mais bien ce qui peut conduire à la persuasion. Son rôle sera donc de «fournir les outils nécessaires au faire-croire de l'orateur, pour que les thèses présentées soient considérées comme justes et pour que l'énonciataire atteigne le croire-être, indépendamment du type de connaissance en question » (italiques de l'original). À la suite d'Aristote, Greimas et Courtès (1993: 423) observent que «le discours vraisemblable n'est pas seulement une représentation correcte de la réalité socioculturelle, mais aussi un simulacre monté pour faire paraître vrai et qu'il relève, de ce fait, de la classe des discours persuasifs » (italique de l'original).

11 Le conte ne dit pas que Janjão a accepté le contrat proposé par le père, puisque la performance - celle consistant à réussir dans la vie moyennant la conjonction avec l'objet de valeur prestige social - est présentée comme une simple possibilité «à venir », bien que l'intérêt manifesté par le fils pour le discours du père et ses propres interventions nous portent à croire que la manipulation se soit effectivement produite et ait instauré chez Janjão un vouloir/un devoir-être médaillon. De toute manière, les instructions données par le père - les comportements qu'on attend d'un médaillon -, fonctionnent comme un ensemble de programmes d'usage (constitution de la compétence modale du sujet, comme nous l'avons déjà signalé), par rapport au programme de base (la performance que constitue la transformation en médaillon). Nous présentons ci-dessous une liste de quelques-uns des programmes d'usage ayant une incidence sur différents domaines :

a : Réaliser des activités susceptibles de réduire l'intellect et ne permettant pas de formuler des idées, telles que s'intéresser à des choses banales (la coupe d'un gilet, les dimensions d'un chapeau); pratiquer certains sports ou jeux, comme le billard, qui ne font pas appel au raisonnement; lire des manuels de rhétorique; se promener dans la rue toujours accompagné par quelqu'un (car la solitude amène à réfléchir) ; fréquenter les librairies (non pour lire mais pour se tenir au courant des potins du jour).

b. Insérer dans sa pratique oratoire des éléments conventionnels, consacrés par l'usage : citations, maximes et expressions toutes faites. Se soumettre à la pensée des autres et valoriser le savoir déjà établi.

c. Parler de la modernité : être au courant en particulier des découvertes, des sujets auxquels la science s'intéresse à ce moment, mais sans aucune intention de mettre celle-ci en pratique. 
d. Ne pas ménager ses efforts pour la publicité et profiter pleinement de celle-ci pour se faire connaître. Se faire inviter aux fêtes, faire en sorte que sa présence y devienne indispensable.

e. Entrer dans la politique et s'affilier à un parti (à condition de n'adopter les idées d'aucun d'entre eux) et utiliser la tribune pour attirer l'attention du public sur sa personne, en évitant toutefois d'aborder des sujets se prêtant au surgissement d'idées nouvelles et en s'orientant vers la «métaphysique politique » (où tout est déjà pensé d'avance).

f. Éviter l'ironie et lui préférer la bonne plaisanterie populaire qui ne requiert pas de grand effort intellectuel, etc.

12 En somme la méthode à suivre pour devenir médaillon consiste à s'en tenir à ce qui est déjà « découvert, formulé, étiqueté, empaqueté » (53) et à fuir tout ce qui, au contraire, pourrait conduire à la réflexion et à l'originalité. L'important est donc de contribuer à la permanence de l'ancien en y prenant appui sur celui-ci pour être reconnu dans une société qui apprécie par-dessus tout la stabilité et l'apparence et qui attache la plus grande importance à l'opinion des autres.

Cela fait resurgir devant nous la question déjà abordée du paraitre vrai, ce qui renvoie à des modalités véridictoires qui, articulées comme catégorie modale en /être/ vs / paraître/, établissent la corrélation entre le plan de l'immanence et celui de la manifestation. Ceci parce que, pour le médaillon, ce qui importe n'est pas l'essence (l'être), mais l'apparence (le paraître). Si nous envisageons l'idée de médaillon comme une distinction - comme quelque chose qui nous permet de nous élever « au-dessus de l'obscurité du commun ", comme le dit le père, nous verrons que, paradoxalement, le "véritable " médaillon est menteur (articulation du paraître avec le non-être) ou, à la limite, faux (articulation du non-être et du non-paraître). D'ailleurs, l'« âme extérieure ", celle que nous donnons à voir de nous-mêmes (aux autres) et qui s'appuie sur l'apparence, réapparaît chez le personnage de Jacobina, de l'autre conte de Machado de Assis, Le miroir, qui sera analysé dans la prochaine section.

Du point de vue discursif, le conte, comme tout texte littéraire, est essentiellement figuratif et construit donc un simulacre du monde. La figure principale est celle du médaillon (présente dès le titre, étant donné que le conte se veut un ensemble d'instructions théoriques destinées à guider la pratique " d'être médaillon »). Éclairant cette figure, nous retrouvons ici le thème de la quête du pouvoir, en rapport avec l'ascension sociale, mais aussi d'autres thèmes qui s'articulent par le biais de l'ironie, au noble et difficile (puisqu'il requiert temps et patience: en moyenne 45 ans) office de médaillon: la futilité, la superficialité, l'ostentation, la répétitivité et enfin la médiocrité.

15 Ainsi, exercer cet « office » - qui n'a rien d'un office, ni rien de noble ni de difficile consiste en réalité à ne rien faire ou, à tout le moins, à ne rien faire qui ait quelque utilité ou importance dans la société. Selon le Robert, l'individu médiocre peut être défini comme "inférieur, faible, qui ne dépasse pas ou même n'atteint pas la moyenne ». Nous voyons donc que le "médaillon complet », comme l'affirme le père, est plus proche des « parasites » qui habitent l'univers machadien que de quelqu'un qui se distingue, qui se fait remarquer parmi ses pairs par des actions importantes ou grandioses.

16 En termes d'ethos, ce que fait le père dans Théorie du médaillon, c'est de proposer à son fils de construire un ethos nul qui, loin d'affirmer une identité, finit par la nier. Selon le 
père, un homme sans qualités aurait plus de chances de progresser dans l'échelle sociale. Cette proposition - celle selon laquelle un homme sans idées serait plus apte à persuader et, ainsi, à obtenir du succès - fascine par son incongruité et renvoie à un soi-disant ethos d'expertise qui sera subverti par l'ironie de l'énonciateur et contribuera de cette manière, comme on le verra, à la construction de son ethos propre (celui de l'énonciateur).

$\mathrm{Si}$, au niveau narratif, la manipulation implique un contrat fiduciaire/véridictoire entre destinateur et destinataire, qui sont actants du texte-énoncé (les personnages du conte), il y a également au niveau discursif un faire persuasif et un faire interprétatif corrélaires, mais instaurés maintenant entre les actants de l'énonciation : énonciateur et énonciataire. Une adhésion fiduciaire est alors nouvellement en jeu, qui implique surtout le croire et qui révèle le caractère idéologique de l'interprétation comme reconnaissance de la vérité dans le discours.

Selon Fiorin (2003: 163-164), il y a basiquement dans le texte trois instances énonciatives hiérarchisées. La première est celle de l'énonciation considérée comme acte implicite de production de l'énoncé (et logiquement présupposée par l'existence même de celui-ci). Cette instance est celle du « je » et du " tu », appelés énonciateur et énonciataire (destinateur et destinataire de l'objet discours) : il s'agit de l'auteur et du lecteur implicites (ou abstraits). Dans la seconde instance de la hiérarchie énonciative, nous avons le narrateur et le narrataire : le «je »et le «tu » installés dans l'énoncé, lesquels peuvent rester implicites (dans le cas où l'histoire est contée à la troisième personne). La troisième instance apparaît quand le narrateur, déjà installé dans le texte-énoncé, donne la parole en discours direct aux personnages. Dans ce cas, le couple je/tu est celui de l'interlocuteur/interlocutaire.

"Théorie du médaillon » se déploie en forme de dialogue, ce qui implique le couple interlocuteur/interlocutaire (personnages qui parlent en discours direct). Dans ce cas, nous avons un narrateur absent, car personne n'assume explicitement le récit : le conte donne l'impression de se raconter lui-même à partir des répliques des personnages. Autre aspect important : le fait que, bien que le « je »et le « tu » soient réversibles, il y a dans le conte prédominance de l'intervention du père, ce qui fait que le dialogue se transforme pratiquement en un monologue: le fils se limite à des répliques plutôt courtes qui impliquent des manifestations d'adhésion ( « Je comprends »), des demandes d'éclaircissement («Si j'entre au Parlement, pourrai-je monter à la tribune ?») ou des commentaires banals (« Fichtre! Que la profession est difficile!»), ce qui lui confère le rôle thématique d'inexpérience et d'ingénuité - rôle renforcé d'ailleurs par le nom même qui le désigne : Janjão -, qui va à l'encontre du rôle thématique de sagesse et de sagacité du père - à quoi s'ajoute naturellement une bonne dose de manque de scrupules, où les moyens - les actions réalisées - justifient les fins : l'obtention d'un statut.

En excluant l'instance narrative et en optant pour le dialogue, l'énonciateur renforce le caractère dramatique du texte, construisant ainsi des effets de réel propres au discours direct. Mais quelle sorte de contrat énonciatif établit-il avec son lecteur? En premier lieu, considérant qu'il s'agit d'un conte (œuvre de fiction), l'énonciataire attend un texte qui soit pour le moins vraisemblable : même si en fait cela n'est pas arrivé, cela aurait pu arriver. Et cet effet est construit au début même du conte : il est normal que les parents, étant donné leur plus grande expérience, donnent des conseils à leurs enfants. Cette « illusion référentielle » est renforcée par la mention d'une date (celle de 
la naissance de Janjão : 5 août 1854) et par l'ancrage dans une situation concrète : une conversation à huis clos, après un dîner à l'occasion de la majorité de Janjão, ce qui conduit à croire que le contrat implicite entre énonciateur et énonciataire a été rempli. Voyons le dialogue :

- Tu as sommeil?

- Non, père.

- Moi non plus. Parlons un peu ; ouvre la fenêtre... Quelle heure est-il ?

- Onze heures.

- Le dernier convive de notre modeste dîner s'est retiré. Alors, garnement, tu as maintenant vingt et un ans. Il y a vingt et un ans, le 5 août 1854, tu voyais le jour, un petit bonhomme de rien du tout, et te voilà un homme, avec de longues moustaches, quelques amourettes...

- Papa...

- Inutile de rougir. Parlons sérieusement, comme deux amis. Ferme cette porte ; je vais te dire des choses importantes [...] (43).

Néanmoins à mesure que la lecture avance, nous constatons que les propos que le père tient à son fils ne pouvaient être assumés par l'énonciateur, car ils soutiennent l'insoutenable, subvertissent complètement les canons de la morale. De ce fait, le contrat de vraisemblance établi - ou apparemment établi - au début est rompu et un conflit est créé entre énoncé et énonciation, ce qui est le propre de l'ironie.

L'ironie, selon Oliveira (1991: 107), se manifeste « dialectiquement dans la présentation des personnages et la distanciation critique adoptée par l'auteur-implicite par rapport à ceux-ci ». Du coup, le jeu entre l'être et le paraître, entre ce que l'on est en réalité et ce que l'on paraît être dans la pratique sociale, aboutit à un « expédient ironique utilisé par l'auteur-implicite qui présente le processus mais ne s'identifie pas avec lui, préférant les coulisses à la scène ». Parmi les multiples passages ironiques qui tissent le conte sur un mode polyphonique, nous en avons choisi un à titre d'illustration. Il s'agit de l'extrait dans lequel le père, abordant la question de la publicité, évoque le cas où des amis offriraient au médaillon un portrait ou un buste :

[...] non seulement les règles de la plus élémentaire politesse t'obligent à accepter le portrait ou le buste, mais il serait malvenu d'empêcher tes amis de l'exposer en quelque lieu public. De cette manière le nom demeure lié à la personne ; ceux qui auront lu ton récent discours (supposons) à la session inaugurale de l'Union des coiffeurs reconnaitront dans la composition des traits de l'auteur de cette œuvre grave, dans laquelle le « levier du progrès » et la « sueur du travail » parviennent à vaincre les « gosiers béants » de la misère. [...] Tu inviteras ensuite tes meilleurs amis, tes parents, et si possible une ou deux personnalités en vue. Mieux encore : si ce jour est un jour de gloire ou de réjouissance, je vois mal comment tu pourrais refuser une place à table aux journalistes. Quoi qu'il en soit, si les obligations de ces messieurs les retiennent ailleurs, tu peux les aider d'une certaine manière, en rédigeant toi-même le compte rendu de la fête... (51).

Il n'est pas difficile d'entendre, sous les propos absurdes du père, la voix mordante d'un énonciateur qui critique certains comportements de la société bourgeoise de l'époque. Quelle pouvait bien être, par exemple, l'importance d'un discours «à la session inaugurale de l'Union des coiffeurs »? Ou que dire de l'éthique quand on agit de manière intéressée ? C'est la preuve de la médiocrité la plus absolue. L'ironie devient donc directement proportionnelle à la banalité des propos tenus par les personnages. De cette manière, l'énonciateur subvertit le contrat de vraisemblance avec l'énonciataire, amenant celui-ci à inverser les paroles de l'interlocuteur-père pour en arriver à son point de vue (celui de l'énonciateur). 

l'identité au moyen du concept d'ethos, c'est-à-dire du caractère attribué à celui qui prend la parole. Si ce concept est relativement commun aux deux penseurs, la manière dont il se constitue chez eux varie de façon considérable. Pour Isocrate, l'ethos est extérieur au discours et a son origine dans l'image publique de l'orateur telle qu'elle est perçue par ceux qui l'écoutent. En d'autres termes, pour l'auteur de Contre les sophistes, l'ethos consiste dans la réputation de l'individu. Or, comme celle-ci ne peut se constituer qu'au moyen de discours appartenant aux genres les plus variés, y compris non-verbaux, nous pouvons conclure que, pour Isocrate, le sujet auquel on attribue un ethos est toujours un « il », quelqu'un dont on parle. De son côté, le discours produit disons par le détenteur de la réputation - sera accepté dans la mesure où il correspondra à son image publique (si celle-ci, bien entendu, est positive). Cette image, comme nous venons de le dire, naît des discours concernant cet orateur.

Par contre, dans la vision d'Aristote, l'ethos est construit par le sujet lui-même quand il utilise la parole. C'est la manière dont il aborde tel thème déterminé, le vocabulaire utilisé, son expression corporelle et faciale, les connaissances dont il fait preuve, enfin toute la gamme de moyens qui seront utilisés au cours du dire et qui confirmeront ou non le dit. En d'autres termes, dans la vision d'Aristote, le dire de l'orateur, en fonction 
de ce qu'il donne à entendre, peut être un instrument fondamental de persuasion beaucoup plus efficace que ce qui est dit directement. Pour ce motif, Barthes (1985: 146) affirme que tout individu, quand il fait usage de la parole, ne se borne pas à transmettre les informations désirées mais dit aussi à travers celles-ci qu'il est ou n'est pas ceci ou cela. D'un autre côté, il est rare que quelqu'un s'attribue un ethos en l'affirmant directement : il faut en donner la preuve. Il est nécessaire en quelque sorte que le public, de lui-même, crée cette image de l'énonciateur. Les sémioticiens diront que l'ethos implique l'énonciation de l'énonciation - et, de fait, l'énonciation est toujours énoncée : un texte scientifique lui-même, produit dans un langage "froid", "objectif", sans marque de première personne, crée en réalité une image de l'énonciation : celle justement d'un énonciateur qui connaît les règles de la science et entend que celle-ci doit être dépourvue de marques de subjectivité. Donc, il est clair que, tout comme dans le cas de la réputation discutée ci-dessus, il s'agit d'une image créée et qu'étant donné l'objectif que nous poursuivons, il importe peu qu'elle corresponde ou non à la réalité.

Si, d'un côté, la construction d'un ethos est, selon la rhétorique, une étape essentielle du processus de persuasion, d'un autre côté, cette construction ne se complète que si le destinataire est capable de reconstituer cet ethos, d'attribuer à celui qui parle tel ou tel caractère, et de lui assigner ainsi une identité. Arrêtons-nous sur cette question.

Selon Ricoeur (1990 : 39), identifier un individu spécifique c'est le reconnaître un à l'intérieur d'une série d'éléments de la même classe. Dans les campagnes électorales par exemple, l'un des objectifs du candidat est de faire en sorte que l'électeur le reconnaisse au milieu d'une multitude de semblables et qu'il le fasse au moyen des prédicats personnels qui le distinguent : nom, parti, compétence et le fait même de se présenter comme un homme commun, égal aux autres. Peu importe ce qui le différenciera, ce qu'il faut c'est qu'il réussisse à se montrer différent des autres, à se montrer unique dans sa classe; enfin qu'il parvienne à s'attribuer une identité, un ethos, quelle que soit son identité réelle. L'identité surgira alors de l'insertion du sujet dans des classes successives, jusqu'à ce qu'il devienne membre de la classe d'un unique individu : lui-même. Nous pouvons observer que, bien que l'identification s'établisse suivant les mêmes schémas que ceux utilisés dans un processus de classification, il existe entre les deux opérations un rapport d'opposition en raison des objectifs visés par l'une et par l'autre. En effet, si la première vise à cerner l'individu au moyen de l'explicitation de ses idiosyncrasies, la seconde vise à lui soustraire ce qui lui est propre pour souligner ce qu'il partage avec les autres et le rattacher à une classe : alors que la première vise les différences, cette dernière s'attache aux ressemblances.

31 Dans la même oeuvre, Ricoeur (1990: 39-54) propose trois mécanismes qui peuvent nous conduire à l'identification d'un individu : la prédication (ou, plus exactement, les descriptions définitoires); les noms (de famille, prénoms); et les indicateurs (ou, plus précisément, les déictiques). Les descriptions définitoires sont celles qui peuvent isoler un unique individu au milieu de sa classe. Conformément à ce qui a été cité plus haut, la classification est utilisée dans un but totalement opposé à celui-ci, non celui de cerner ce qui est unique, mais de chercher ce qui est collectif ; par exemple, à l'intérieur de l'ensemble d'êtres humains du sexe féminin et d'âge avancé (critère de constitution d'une classe), on en recherche un unique, le plus âgé, processus qui conduit, pour l'identifier, à déterminer l'unicité de l'individu. 
rôle fondamental. En général, la description définitoire comprend un élément quelconque qui renvoie directement ou indirectement au corps : couleur des cheveux, poids, âge... Naturellement, il est possible de décrire quelqu'un sans faire appel à ces caractéristiques, mais ce serait plus compliqué du point de vue opérationnel. La participation du corps n'est pas non plus sans importance dans le second mécanisme (l'adoption de noms et de numéros), car en général les documents comportent, outre le code, une photographie du sujet : c'est l'image du corps qui permet d'établir en toute sécurité un rapport entre un code et un sujet. Enfin, les déictiques sont porteurs de l'idée, implicite, de localisation physique du sujet dans le temps et dans l'espace. Par exemple, les pronoms celui-ci, celui-là ; ils se réfèrent à la distance plus au moins grande ou petite entre le sujet qui parle et le corps auquel il est fait référence. Il est évident que cette distance ne peut être estimée que si la position dans l'espace du corps des personnes concernées nous est donnée. Comme le fait observer Ricoeur, le corps est un élément fondamental pour la définition d'une identité ; pour cette raison, il est considéré comme un particulier de base, c'est-à-dire un élément primordial pour la définition de l'identité. Le corps enfin n'est pas seulement présent dans les mécanismes cités de définition de l'identité, mais est aussi un élément important dans la définition de l'ethos: il fonctionne en quelque sorte comme un articulateur entre celui-ci (l'ethos) et celle-là (l'identité).

La définition d'un ethos embrasse les trois opérations citées, selon la conception que nous avons à l'esprit. Pour Isocrate, l'ethos correspond à une description définitoire du caractère de l'individu, une description qui, dans un second moment, est rapportée à 
un nom, de manière à établir un lien entre les deux et à constituer ainsi la réputation du sujet. Comme nous pouvons l'observer dans ce cas, l'ethos est vu comme un acte de prédication, relatif au «il» dont on parle. D'un autre côté, dans la conception d'Aristote, selon laquelle l'ethos est construit par le discours, une série de prédicats est aussi attribuée à l'orateur (honnêteté, compétence, etc.). Néanmoins cette attribution n'est pas faite par de tierces personnes, mais au moyen du discours proféré par le sujet lui-même. Dans ce cas, le lien entre prédication et sujet ne s'établit pas au moyen d'un nom ou d'un code mais par la référence identifiante, laquelle permet de rapporter le dit au dire et donc à l'acteur de l'énonciation. En l'absence de ces marques référentielles, l'énonciation ne pourrait être énoncée et, sans ces marques, la constitution de l'ethos serait impossible, puisque celui-ci se rapporte justement à l'énonciation.

Ces questions sont discutées par Machado de Assis dans Le miroir, l'un de ses contes les plus célèbres. Celui-ci se distingue non seulement par son style soigné et par l'intrigue elle-même, mais aussi par son sujet. En effet, dans Le miroir, récit fictionnel et théorie se confondent en quelque sorte et révèlent que la distance qui les sépare est moindre qu'on ne le pense à première vue dans la mesure où l'un et l'autre dépendent de l'habileté de l'énonciateur à créer des effets de vérité. Cependant, avant d'examiner le texte, il convient de faire sur son titre une brève réflexion. Les dictionnaires nous indiquent qu'un miroir est essentiellement une surface qui réfléchit des images. D'un autre côté, le verbe "réfléchir" a deux acceptions différentes, mais également intéressantes. Le Petit Robert affirme que "réfléchir» peut aussi bien signifier " renvoyer par réflexion dans une direction différente ou dans la direction d'origine " que «faire usage de la réflexion, c'est-à-dire opérer un retour de la pensée sur ellemême en vue d'examiner plus à fond une idée, une situation, un problème ». Autrement dit, la fonction exercée par le miroir est de renvoyer images et pensées à leur point d'origine, dans notre cas, à l'énonciation. Bien qu'une telle idée soit étrangère au concept de miroir qui prédomine dans notre esprit, nous pouvons voir qu'elle est sousentendue dans les définitions présentées aussi bien par le Robert que par le Littré:

Miroir

Littré : Ce qui représente une chose et la met pour ainsi dire sous nos yeux.

Robert : Ce qui offre à l'esprit l'image, la représentation des personnes, des choses, du monde.

En réunissant ces deux définitions, nous pourrions dire que la fonction exercée par le miroir permet à l'observateur de s'arrêter sur ce qu'il voit, soit parce que le miroir représente quelque chose qu'il met sous ses yeux, soit parce qu'il offre cette représentation à son esprit. Ce qui est toutefois plus important, c'est le fait que, dans le conte en question, le miroir remplit rigoureusement ces deux fonctions : d'un côté, il fait revenir l'image à son point d'origine, c'est-à dire qu'il fournit au sujet une image de lui-même et, d'un autre côté, il amène le sujet à examiner une idée, un problème qui concerne son propre corps, donc son identité même, comme nous venons de le voir. Pour reprendre le titre du livre de Ricoeur Soi-même comme un autre, le miroir est un objet qui permet au sujet de se voir lui-même comme tel, comme s'il voyait un autre, lui offrant ainsi une image de lui-même qu'il n'aurait pas autrement.

Mais revenons au conte. Le personnage principal, Jacobina, formule une théorie selon laquelle l'homme abrite non pas une âme, mais deux : l'une qui regarde du dedans vers le dehors et l'autre qui regarde du dehors vers le dedans. Ce simple énoncé met déjà en lumière le fait que la matière du récit a un rapport avec la théorie qui a été discutée jusqu'ici et que la question de l'identité est présente tout au long du récit. Essayons 
alors d'en savoir un peu plus sur Jacobina qui, dans ce conte, assume lui-même une grande partie du récit.

Le narrateur premier raconte que Jacobina avait "entre quarante et cinquante ans", que c'était «un rentier, originaire de province, [...] un homme intelligent, non dépourvu d'instruction et d'esprit, semble-t-il, astucieux et caustique. Il ne prenait jamais part aux discussions ... » (55). Ces brèves lignes nous fournissent une ébauche de l'identité du personnage, laquelle se construit en s'inscrivant dans des classes de plus en plus restreintes, jusqu'à ce qu'on arrive à une classe réduite à un seul élément : Jacobina lui-même. Nous percevons en outre quelques autres prédicats plus spécifiques qui définissent son caractère, et donc son ethos, et une manière d'être dans le monde : il est astucieux, caustique, opposé aux discussions. Cependant l'identité, le «je », donc l' ethos, qui émerge des paroles du narrateur est avant tout un «il», c'est-à-dire quelqu'un dont on parle et auquel sont attribués des prédicats, et non un "soi", l'expression d'une individualité oeuvre de l'individu lui-même quand il s'exprime. C'est un fait que, se fondant sur ces informations, le lecteur peut commencer à construire un ethos donné du personnage et qui, éventuellement, sera ou non confirmé par la manière dont celui-ci s'exprime. Et, de fait, dans les lignes suivantes, Jacobina propose l'existence de deux âmes, ce qui s'oppose d'emblée aux postulats de la religion et de la philosophie. Or, pour la religion, au moins pour le christianisme, à chaque individu correspond une et seulement une âme dont la destinée éternelle dépend de la rectitude de son caractère ou peut-être, en forçant un peu le trait, de son ethos. Pour la philosophie, nous venons de le voir, l'identité elle aussi se constitue au moyen de la détermination d'unicité qui caractérise l'individu. Il est évident qu'il ne peut exister d'individualité sans unicité, cela non pour des raisons métaphysiques mais pour des raisons logiques : l'individualité présuppose l'unicité.

L'identité présuppose aussi la permanence et la constance de l'être, la conscience de soi-même, et un nombre limité de prédicats. En conséquence, la thèse de Jacobina est pour le moins polémique : un fait qui finira par caractériser son énonciateur comme quelqu'un de même nature, une information qui n'avait pas été fournie par le narrateur premier, lequel n'a pas présenté Jacobina comme quelqu'un de polémique, mais au contraire comme quelqu'un qui évite les discussions, une donnée qui est confirmée au moment où Jacobina est contesté par son interlocuteur et préfère lui donner raison.

41 Néanmoins, à côté de cette caractéristique, il en surgit une autre au sujet de laquelle le narrateur premier n'avait rien affirmé : Jacobina est également autoritaire, comme l'indiquent ses paroles au moment où il introduit son récit: «je n'admets pas la réplique. Quoi que vous m'objectiez, je termine mon cigare et pars sur-le-champ me coucher » (57). Les verbes " admettre », « terminer »et " aller » à la première personne, ne permettent aucunement de douter que le dire doit être attribué à Jacobina qui, en raison du contenu du dit, en vient à acquérir un ethos différencié, celui de quelqu'un d'autoritaire. Ainsi, à côté des caractéristiques qui lui sont attribuées par le narrateur premier quand il décrit Jacobina (comme un « il »), nous en voyons surgir une autre qui naît de son propre discours (dans lequel il est vu comme un «je ») : Jacobina non seulement confirme l'image qu'a de lui le narrateur, mais en ajoute une autre : celle de quelqu'un qui n'admet pas qu'on le conteste. D'un autre côté, sa manière de narrer et le contenu même de sa narration - qui sont deux composantes importantes de l'ethos viennent confirmer qu'il est de fait, "astucieux, caustique et non dépourvu d'instruction ", sans toutefois nous permettre de confirmer s'il est riche ou homme de 
la province. En bref, deux principes de construction de l'identité sont en jeu: d'une part, comme prédication quand le narrateur dresse la liste des prédicats de Jacobina et, d'autre part comme « référence identifiante ", quand Jacobina lui-même nous permet, au moyen de son propre discours, de connaître ses prédicats. Les deux principes se complètent l'un l'autre.

Cependant, le conte met en jeu un troisième principe de constitution de l'identité, expliqué lui aussi par la sémiotique et discuté par Ricoeur : l'histoire ou le parcours d'un sujet aux différentes étapes de sa vie. De fait, comme l'affirme le philosophe, l'identité d'un sujet se construit tout au long de sa vie par le biais des tâches qu'il exécute, qui font qu'il change tout en restant égal à lui-même. D'un autre côté, pour la sémiotique, l'identité se constitue à partir des objets avec lesquels le sujet se trouve en rapport aux divers moments de sa vie. Mais revenons à la théorie de Jacobina. Il explique que "l'âme extérieure » du sujet (celle du regard du dehors vers le dedans) peut être un nombre infini de choses: un hochet d'enfant, un livre, le pouvoir, l'argent... Mais ce n'est pas tout. Cette « âme extérieure » peut elle-même changer, dans certains cas plusieurs fois, et la perte de cette âme extérieure équivaudrait à la perte de la moitié de l'âme, donc de l'existence. L'idée peut paraître étrange, mais trouve place dans la sémiotique, car, selon cette science, le sujet n'acquiert l'existence qu'en raison des objets avec lesquels il est en jonction; si ces objets changent, le sujet change lui aussi. Et voici que la théorie de Jacobina nous fait revenir à la sémiotique. Mais ce n'est pas tout. Jacobina affirme que cette « âme extérieure » peut changer et que le mobile de ce changement de l'âme, c'est l'objet visé maintenant par le sujet, ou plutôt les valeurs qu'il y voit investies, car ce n'est par l'objet en soi qui l'intéresse mais la valeur dont cet objet est un simple support. Chaque fois que le sujet entre en conjonction avec un objet de valeur différent, il changerait, au moins partiellement d'identité, et donc d'âme extérieure. Son existence équivaudrait ainsi au total des différents objets de valeur avec lesquels le sujet est ou a été en conjonction. Or la définition de parcours narratif du sujet n'est rien d'autre que la somme d'états (de conjonction ou de disjonction) qu'assume le sujet par rapport à ses objets.

Jacobina explique qu'il avait changé d'âme en devenant sous-lieutenant de la garde nationale. À partir de sa nomination, il était devenu objet de toute l'attention des membres de sa famille et de ses amis, qui s'étaient mis à le traiter avec la plus grande déférence. Et pourtant, au Brésil, la garde nationale exerçait, dans la plupart des cas, une fonction purement décorative. Appartenir à ses cadres n'avait pas d'importance, mais la valeur qu'attribuaient parents et amis de Jacobina à cette nomination les amenait à lui adresser leurs éloges pour sa nouvelle position. Leur attitude sera fondamentale pour la suite du récit. En termes sémiotiques, l'identité de Jacobina change, quand il se trouve en conjonction avec l'objet de valeur "prestige social» investi dans l'objet "sous-lieutenant de la garde nationale", sous la figure de l'uniforme. Autre indicateur de changement d'identité qui s'est produit chez Jacobina : l'altération de son nom. Au lieu de l'appeler Joãozinho ${ }^{7}$ comme le faisaient jusqu'alors parents et amis, on lui donne du «M. le sous-lieutenant »: nous voyons ici par ailleurs l'importance que prend le nom dans le processus d'identification. En bref, toutes les caractéristiques qui le définissaient jusque-là sont éliminées et, en leur lieu et place, s'en installent d'autres, qui deviennent à leur tour constitutives de son identité.

L'importance de ce fait se voit confirmée dans la seconde partie du conte, où Jacobina se retrouve tout seul dans la ferme de sa tante, cette dernière étant en voyage et les 
esclaves en fuite ${ }^{8}$. La solitude introduit le silence et congédie la notoriété qui jusque-là avait entouré le jeune sous-lieutenant. C'est la fin des marques de respect et de déférence auxquelles il s'était habitué depuis sa nomination. La solitude peu à peu inquiète Jacobina. L'inquiétude engendre l'anxiété, et celle-ci la panique. À un moment donné, le jeune homme se regarde dans le miroir qui lui renvoie l'image évanescente de son corps, « ombre d'ombre ». Désespéré, il lui vient à l'esprit une idée "salvatrice »: revêtir de nouveau son uniforme, qui était motif de fierté générale, mais qu'il avait cessé d'utiliser. L'idée produit l'effet imaginé et il se voit parfaitement dans le miroir sans le moindre trouble. Avec le retour de l'identité que l'uniforme lui apporte, le corps physique revient lui aussi, attestant l'imbrication mutuelle de l'identité et du corps, comme le propose Ricoeur.

L'explication ne présente aucune difficulté. L'âme extérieure de Joãozinho (souvenonsnous que c'était là son nom avant sa nomination à la garde nationale) redevient son grade de sous-lieutenant. Or, comme tous - fermière et esclaves - sont partis, il n'y a plus personne pour exalter sa promotion, et voilà que sa nouvelle âme le quitte. L'intérêt suscité par le récit grandit quand la dégradation de l'identité, causée par l'absence d'admirateurs, atteint un degré tel qu'elle entraîne la disparition de son corps, élément primordial (comme nous l'avons vu) dans la définition de l'identité de l'individu. Cette fois encore, nous percevons l'importance des postulats de la sémiotique car ce n'est pas seulement de lui-même que le sujet tient sa valeur mais des objets avec lesquels il est en conjonction; de leur côté ces objets ne tirent pas leur valeur de ce qu'ils sont eux-mêmes, mais des valeurs qui y sont investies. Pour autant, Jacobina n'a pas cessé d'être sous-lieutenant mais «être sous-lieutenant » a cessé d'être une valeur, car se voyant seul, Jacobina n'a plus eu personne pour lui adresser des éloges pour son poste. Les conséquences se font sentir sans tarder. En effet, comme l'enseigne la sémiotique, le sujet sans objet n'a plus d'identité et la perte de son identité acquiert chez Jacobina une telle dimension qu'il n'arrive plus à se voir dans le miroir : son corps perd de sa densité. Si nous prenons en considération le fait que le corps est l'un des éléments de base au moyen duquel le sujet peut être reconnu, nous verrons que sa disparition correspond à la disparition de l'individu ou au moins de son identité. La solution qui lui vient à l'esprit est ingénieuse : refigurer les valeurs de prestige en revêtant l'uniforme et compenser l'absence d'admirateurs en se tournant vers le miroir qui, par le fait même de lui restituer son image, lui procure finalement un unique admirateur, lui-même. Le fait que la stratégie atteigne son but confirme simplement l'importance du rôle que joue le corps dans ce processus, comme nous l'avons vu quand nous l'avons considéré comme un particulier de base.

Dans une formulation heureuse, Amossy (1999) définit l'ethos comme l'image de soi que le sujet construit dans son discours. De cette façon, l'auteure met en lumière que l'ethos a une contrepartie plus visuelle, figurative et indirectement liée au corps physique puisqu'il s'agit d'une image de soi. Pour ce qui en est du conte de Machado de Assis, disons qu'il fait le chemin inverse en montrant que cette image ne peut être dissociée d'une identité et que celle-ci est le résultat des objets avec lesquels le sujet établit un rapport, l'un des postulats essentiels de la sémiotique. Mais Machado va plus loin et, au moyen d'un récit, montre combien le corps dépend de l'identité et la définit. 


\section{Conclusion}

Cet article a analysé deux contes assez différents l'un de l'autre - ce qui a demandé d'emprunter des voies d'analyse elle aussi distinctes. Toutefois, malgré les différences entre les récits, deux points les rapprochent: tous deux questionnent la notion d'identité et le font en associant, quoique de manière implicite, identité et ethos.

Comme nous l'avons vu, dans Théorie du médaillon, le père propose à son fils de construire un ethos nul, qui finit par nier sa propre identité. Dans Le miroir, nous avons affaire à un processus en quelque sorte opposé : la constatation qu'il n'existe pas une identité mais deux, l'une intérieure et l'autre extérieure, cette dernière étant changeante de par sa nature même et variant suivant les objets avec lesquels le sujet entre en rapport de jonction (en termes sémiotiques). De toute manière, avoir différentes identités équivaut à n'en avoir aucune, et là nous revenons à une situation semblable à celle du premier conte.

Cependant, outre le point d'arrivée, quelque chose de plus rapproche les deux récits : le processus par lequel, dans un cas, s'annule l'identité, et, dans l'autre, le sens. Le père de Janjão propose à celui-ci d'utiliser des figures de rhétorique déjà usées et dépourvues de sens, cela pour se créer une identité - exactement le contraire de ce que proposait Isocrate dans Contre les sophistes. D'un autre côté, selon le cas de Jacobina, la multiplicité d'identités découle de la valeur attribuée aux objets avec lesquels le sujet établit un rapport. Et, sur ce point, Jacobina réaffirme les postulats de la sémiotique.

En synthèse, les deux contes de Machado de Assis nous ont permis de réfléchir a diverses questions liées soit à la problématique de l'ethos et de l'identité, soit à d'autres problématiques liées aux moyens de persuasion mis en œuvre dans le rapport entre les personnages ainsi qu'entre l'auteur et le lecteur implicites (énonciateur / énonciataire). Dans ces cas, les modèles sémiotiques sont peu à peu "appelés" à la rescousse, confirmant la productivité du « dialogue » établi entre les deux disciplines.

\section{BIBLIOGRAPHIE}

Amossy, Ruth (éd.). 1999. Images de soi dans le discours. La construction de l'ethos (Lausanne :

Delachaux \& Niestlé)

Aristote. 1991. Rhétorique. Trad. C. E. Ruelle (Paris : Livre de poche)

Assis, J-M. Machado de. 2002. La théorie du médaillon et autres contes. Trad. Florent Kohler (Paris : Métaillé)

Barros, Diana L. P. de. 1990. Teoria semiótica do texto (São Paulo : Ática)

Bordron, Jean-François, Jacques Fontanille et al. 2000. « Sémiotique du discours et tensions rhétoriques » (= Langages 137)

Cruz, Dilson F. da. 2009. O éthos dos romances de Machado de Assis (São Paulo : EDUSP) 
Ducrot, Oswald. 1987. O dizer e o dito (Campinas, SP : Pontes)

Fiorin, J. Luiz. 2003. « Pragmática », Fiorin, J. Luiz. (org.) Introdução à linguística II (São Paulo :

Contexto)

Greimas, Algirdas J. 1983. Du sens II (Paris : Seuil)

Greimas, Algirdas J. \& Joseph Courtés. 1979. Sémiotique, dictionnaire raisonné de la théorie du langage

I (Paris : Hachette)

Houaiss, Antônio. 2009. Dicionário da língua portuguesa (Rio de Janeiro : Objetiva)

Maingueneau, Dominique. 2001. Análise de textos de comunicação (São Paulo : Contexto)

Maingueneau, Dominique. 2006. Cenas da enunciação (Curitiba, PR : Criar Edições)

Maingueneau, Dominique. 2008. «A propósito do ethos », Motta, Anna Raquel \& Luciana Salgado

(orgs.). Ethos discursivo (São Paulo : Contexto), 11-29

Oliveira, Edson S. de. 1991. «Machado de Assis : representação e ironia em "Teoria do

medalhão" ", Artimanhas da ironia 11, n 13 (Belo Horizonte : Centro de Estudos Portugueses)

Ricoeur, Paul. 1990. Soi même comme un autre (Paris : Seuil)

\section{NOTES}

1. Voir Bordron, Fontanille et al. 2000.

2. Pour l'analyse des deux contes, nous avons examiné l'édition française de 2002.

3. NDT : Janjão : surnom à la fois affectueux et moqueur. Augmentatif du prénom João.

4. NDT: Medalhão [médaillon]: (sens figuré) individu important, personnage en vue, professionnel de renom; (figuré péjoratif) individu parvenu à des responsabilités importantes mais sans avoir les qualités requises pour la fonction, d'après le Dicionário da língua portuguesa, de A. Houaiss.

5. Le programme narratif est considéré comme l'unité élémentaire opératoire de la syntaxe narrative. Il se situe à fois dans la dimension pragmatique et dans la dimension cognitive de la narrativité, entendue comme transformation d'états.

6. Cet aspect a été repris dans les principaux travaux contemporains en France. Voir Ducrot (1987), Maingueneau (2006; 2008) et Amossy (1999).

7. NDT : Joãozinho: diminutif du prénom João.

8. Le conte se passe à une époque antérieure à l'abolition de l'esclavage au Brésil, proclamée le 13 mai 1888.

\section{RÉSUMÉS}

Le présent article se propose d'établir un dialogue productif entre la sémiotique du discours (ou greimassienne) et la rhétorique, en examinant deux contes de l'écrivain brésilien Joaquim Maria Machado de Assis (1839-1908) intitulés Teoria do medalhão ["Théorie du médaillon »] et 0 espelho [«Le miroir »]. Ces deux textes, très différents dans leur forme et leur contenu, présentent un 
point commun: ils discutent un processus de "déconstruction d'identité». Ainsi, dans le premier, un père se propose d'amener son fils à adopter un comportement et un discours vides dépourvus, donc, d'identité - pour atteindre le prestige dans une société d'apparences. Dans le second, nous assistons à un processus involontaire de perte d'identité, ce qui extrapole des aspects discursifs pour s'emparer du sujet, en menaçant son existence même.

This paper aims to promote a productive dialogue between French Semiotics (Greimas) and Rhetoric, by analyzing two short tales by the Brazilian writer Joaquim Maria Machado de Assis (1839-1908), entitled Teoria do medalhão ["Theory of the medalhão"] and O espelho ["The mirror"]. Though very different in form and content, the two texts share a common point: both of them discuss some kind of "identity deconstruction" process. Thus, in the first tale, a father proposes to teach his son how to adopt an empty - and therefore devoid of identity - discourse and behavior so as to reach success in a society of appearances. In the second tale, we observe an involuntary process of identity loss, which goes beyond discourse and takes possession of the individual subject, thereby threatening his very existence.

\section{INDEX}

Keywords : de Assis (Joaquim Maria Machado), ethos, identity, literature, rhetoric, semiotics Mots-clés : de Assis (Joaquim Maria Machado), ethos, identité, littérature, rhétorique, sémiotique

\section{AUTEURS}

\section{GLAUCIA MUNIZ PROENÇA LARA}

Universidade Federal de Minas Gerais

\section{DILSON FERREIRA CRUZ}

Universidade Presbiteriana Mackenzie 